\title{
Bioactivity of Marine Organisms: Part XI- Screening of some Marine Flora from the Indian Coasts $* *$
}

\author{
V. Lakshmi*, A. K. Goel, M. N. Srivastava and R. Raghubir \\ Central Drug Research Institute, Lucknow- 226 001, India
}

\begin{abstract}
With a view to explore of finding the new molecules with therepeutic efficacy for human use, the alcoholic extracts of 33 indentified species of marine flora, collected from Indian coasts, were prepared and screened for a wide range of biological activities. Of these, 1 extract showed antifilarial activity, 1 showed antiamoebic effect and the 1 showed antitrichomonas activity.
\end{abstract}

Key words: Marine flora, Ethanol extracts, Broad biological screening, 3 active extracts.

\section{Introduction}

A programme for the screening of marine flora and fauna extracts of the Indian coasts was started about 20 years ago at this Institute. So far about 350 marine flora and fauna species have been screened for their bioactivity. It is intened to publish the data collected in this programme from time to time, as series of papers, with a view to explore the possibilities of finding new molecules, with proven therapeutic efficacy for human use. The results of biological screening of about 350 marine organisms, mainly collected by National Institute of Oceanography, Goa (India) and Central Drug Research Institute, Lucknow (India) have been reported Bhakuni et al 1992; Dhawan et al 1993; Kamat et al 1981, 1991, 1992; Naik et al 1989, 1990; Naqvi et al 1980; Siddhanta et al 1997 and Lakshmi et al 2006a, 2006b. The present communication deals with the biological activites of the extracts of 33 marine plant species. The biological screening includes tests for antibacterial, antifungal, antitrichomonas, antiprotozoal, anthelmintic, antiviral, antifertillity, hypoglycemic, effects on cardiovascular system and central nervous system (including gross behavior), effect on isolated tissues, diuretic and anti-inflammatory activities.

\section{Materials and Methods}

\section{Collection of the marine flora}

Under the National Project "Development of Potential Drugs from the Ocean", marine floras were collected from the coasts of Goa, Maharashtra, Tamil Nadu, Gujarat, Orissa and Andaman and Nicobar Islands of India during pre-and post-

*Corresponding author: E-mail: vijlakshmius@yahoo.com

**CDRI communition number 7400 monsoon periods. The material was rinsed with water to free them from extraneous matter, shade dried.

\section{Preparation of the extracts}

The air dried and powdered material was filled in glass percolators, extracted with $95 \%$ ethanol at room temperature by percolation. The first extract was collected after two days. The process was repeated five times. The solvent from the combined percolates was removed under reduced pressure below $40^{\circ} \mathrm{C}$. The extract was finally dried in a vacuum desiccator and tested for a wide range of pharmacological activities, while a few of them were screened for antileishmanial, antitubercular and anthelmitic activities. The details of these tests have been described earlier Bhakuni et al 1969, 1971; Dhar et al 1968 and Dhawan et al 1977. The experiments were performed after necessary approval of IACC of the Institute.

\section{Results and Discussion}

In Table I, the marine floras have been listed alphabetically, with collection place, date of collection, parts of the parts used and family. The results of the biological screening of the crude extracts, found active in the primary biological screening, are also indicated. Among active extracts, 1 showed artifilarial activity out of 26 tested, 1 showed antiamoebic activity out of 11 tested while 1 extract exhibited anti-trichomonas activity out of 28 tested. 


\section{Table I. List of marine flora screened, place and time of collection and summary of results of biological tests}

Codes for plant parts: [FR: Fruits, LF: Leaves; PL: Entire plant; PX: Entire plant without root; RB: Root bark; ST: Stem] Codes for biological tests: [ A. Antibacterial ( $\mathrm{A}_{2}$ Staphylococcus aureus; $\mathrm{A}_{4}$ Escherichia coli; $\mathrm{A}_{6}$, Mycobacterium tuberculosis $\mathrm{H}_{37} \mathrm{R}_{\mathrm{v}} ; \mathrm{A}_{9}$, Streptococcus faecalis, $\mathrm{A}_{10}$, Klebsiella pnuemoniae; $\mathrm{A}_{11}$, Pseudomonas aeruginosa); $\mathrm{B}$. Antifungal ( $\mathrm{B}_{1}$, Candida albicans: $\mathrm{B}_{2}$, Cryptococcus neoformans; $\mathrm{B}_{3}$, Trichophyton mentagfrophytes; $\mathrm{B}_{6}$.) Aspergillus fumigatus; $\mathrm{B}_{7}$, Sporotrichum schenckiii, $\mathrm{B}_{14}$, Candia parapsilosis (ATCC-22019)); C. Antiprotozoal $\mathrm{C}_{1}$, Entamoeba histolytica strain STA; $\mathrm{C}_{4}$, Plasmodium berghei; $\mathrm{C}_{5}$, Plasmodium falciparum; $\mathrm{C}_{6}$, Giardia lamblia); D, Anihelmenthic ( $\mathrm{D}_{8}$ Antifilarial against Brugia malayi) Antiviral ( $\mathrm{E}_{6}$, Hdrpes) simplex virus; $\mathrm{E}_{8}$, Influenza virus; $\mathrm{E}_{9}$, Japanese $\mathrm{B}$ Encephalitis virus; $\mathrm{G}$. Antifertility $\mathrm{G}_{2}$, Antiimplantation in rats; $\mathrm{G}_{3}$, Spermicidal, $\mathrm{G}_{14}$, Anti-trichomonas; $\mathrm{H}$. Hypoglycemic $\mathrm{H}_{1}$, Glucose loaded rat model; $\mathrm{H}_{2}$, Streptozotocin inuced rat model; J. Effect on cardiovascular system $\mathrm{J}_{2}$, (Effect on blood pressure; J9, Anit-thrombotic), $\mathrm{J}_{10}$, Anti-ischemic; L. Effect on central nervous system ( $\mathrm{L}_{1}$, Gross effects); R. Hypolipidemic $\mathrm{R}_{1}$, Anti-cholesterolemic in triton treated rat model and Y Anti-obestiy ( $\mathrm{Y}_{1}$, Appetite Suppressant)].

\begin{tabular}{|c|c|c|c|c|}
\hline $\begin{array}{l}\text { Botanical Name (Part) } \\
\text { (Family) }\end{array}$ & $\begin{array}{l}\text { Locality and } \\
\text { State }\end{array}$ & $\begin{array}{l}\text { Month and } \\
\text { Year of } \\
\text { Collection }\end{array}$ & $\begin{array}{l}\text { Biological Tests } \\
\text { Conducted }\end{array}$ & $\begin{array}{l}\text { Activity } \\
\text { Observed }\end{array}$ \\
\hline $\begin{array}{l}\text { Aglaia cucullate (Roxb.) Pellegrin } \\
\text { (PX) } \\
\text { Meliaceae }\end{array}$ & $\begin{array}{l}\text { Paradeep } \\
\text { (Orissa) }\end{array}$ & Nob. 2003 & $A_{2,4,6,9-11,} B_{1-3,6,7,14}, E_{9}, G_{2,3}, H_{1}, L_{1}$ & - \\
\hline $\begin{array}{l}\text { Barringtonia racemosa Roxb. } \\
\text { (FR) } \\
\text { (Barringtoniaceae) }\end{array}$ & $\begin{array}{l}\text { South Andaman } \\
\text { (A.\& N. Islands) }\end{array}$ & Mar. 2003 & $A_{2,4,6,9-11} B_{1-3,6,7,14}, D_{8}, E_{6,9}, G_{2,3}, J_{9,10}$ & - \\
\hline $\begin{array}{l}\text { Barringtonia racemosa Roxb. } \\
\text { (PX) } \\
\text { (Barringtoniaceae) }\end{array}$ & $\begin{array}{l}\text { South Andaman } \\
\text { (A. \& N. Islands) }\end{array}$ & Mar. 2003 & $A_{2,4,6,9-11} B_{1-3,6,7,14}, E_{9,} G_{2,3} H_{1,} L_{1}$ & - \\
\hline $\begin{array}{l}\text { Bruguiera sexangula (Lour.) Poir. } \\
\text { (PX) } \\
\text { (Rhizophoraceae) }\end{array}$ & $\begin{array}{l}\text { South Andaman } \\
\text { (A. \& N. Islands) }\end{array}$ & Dec. 2005 & $\mathrm{D}_{8,} \mathrm{E}_{9,}, \mathrm{G}_{2,3}, \mathrm{H}_{1}, \mathrm{R}_{1}$ & - \\
\hline $\begin{array}{l}\text { Brugueira cylindrical (L.) BI } \\
\text { (PX) } \\
\text { (Rhizophoraceae) }\end{array}$ & $\begin{array}{l}\text { Chidambaram } \\
\text { (Tamil Nadu) }\end{array}$ & Jun. 2002 & $\begin{array}{l}A_{2,4,6,9-11} B_{1-3,6,7,14,} C_{1,5}, E_{6,9} \\
G_{2,} H_{1}, L_{1}, R_{1}\end{array}$ & - \\
\hline $\begin{array}{l}\text { Caesalpinia bonduc Roxb. } \\
\text { (PX) } \\
\text { (Caesalpiniaceae) }\end{array}$ & $\begin{array}{l}\text { South Andaman } \\
\text { (A. \& N. Islands) }\end{array}$ & Dec. 2005 & NONE & - \\
\hline $\begin{array}{l}\text { Caulerpa scalpelliformis (R. Br.) } \\
\text { Web. V. Bosse } \\
\text { (PL) } \\
\text { (Caulerpaceae) }\end{array}$ & $\begin{array}{l}\text { Ramnad } \\
\text { (Tamil Nadu) }\end{array}$ & Nov. 2000 & $\begin{array}{l}A_{2,4,6,9-11}, B_{1-3,6,7}, C_{1,4,6} \\
G_{2,3,14} H_{1}, L_{1}, R_{1}\end{array}$ & - \\
\hline
\end{tabular}

Contd. 
Table I. To be contd.

\begin{tabular}{|c|c|c|c|c|}
\hline $\begin{array}{l}\text { Caulerpa scalpelliformis (R. Br.) } \\
\text { Web. V. Bosse } \\
\text { (PL) } \\
\text { (Caulerpaceae) }\end{array}$ & $\begin{array}{l}\text { Ramnad } \\
\text { (Tamil Nadu) }\end{array}$ & Nov. 2002 & $\mathrm{~A}_{2,4,6,9-11}, \mathrm{~B}_{1-3,6,7,14}, \mathrm{E}_{9,}, \mathrm{G}_{2,3}, \mathrm{H}_{1,} \mathrm{~L}_{1}$ & - \\
\hline $\begin{array}{l}\text { Cerbera manghas L. } \\
\text { (FR) } \\
\text { (Apocynaceae) }\end{array}$ & $\begin{array}{l}\text { South Andaman } \\
\text { (A \& N Islands) }\end{array}$ & Mar. 2004 & $\begin{array}{l}A_{2,4,6,9-11} B_{1-3,6,7,14}, E_{9} \\
G_{2,3}, H_{1}, J_{9,10}, R_{1}\end{array}$ & \\
\hline $\begin{array}{l}\text { Cerbera manghas L. } \\
\text { (PX) } \\
\text { (Apocynaceae) }\end{array}$ & $\begin{array}{l}\text { South Andaman } \\
\text { (A \& N Islands) }\end{array}$ & Mar. 2004 & $\mathrm{~A}_{2,4,9-11,}, \mathrm{~B}_{1-3,7,14,} \mathrm{E}_{9,}, \mathrm{G}_{2,3}, \mathrm{H}_{1,2}$ & \\
\hline $\begin{array}{l}\text { Chaetomorpha torta (Farlow) MC } \\
\text { Clatchie } \\
\text { (PL) } \\
\text { (Cladophoraceae) }\end{array}$ & $\begin{array}{l}\text { Ramand } \\
\text { (Tamil Nadu) }\end{array}$ & Jul. 2001 & $\begin{array}{l}A_{2,4,9-11,} B_{1-3,6,7,14,} C_{1,6} D_{8} \\
E_{8,9}, G_{2,3}, H_{1}, J_{2,} R_{1}\end{array}$ & $\mathrm{C}_{1}$ (Antiamoebic) \\
\hline $\begin{array}{l}\text { Chondrococcus hornemanii } \\
\text { (Mert.) Schmitz } \\
\text { (PL) } \\
\text { (Rhizophyllidaceae) }\end{array}$ & $\begin{array}{l}\text { Ramnad } \\
\text { (Tamil Nadu) }\end{array}$ & Feb. 2006 & $\mathrm{~A}_{6,}, \mathrm{~B}_{1-3,6,7,14} \mathrm{G}_{2,} \mathrm{~J}_{9}$ & - \\
\hline $\begin{array}{l}\text { Cynometra ramiflora L. } \\
\text { (FR) } \\
\text { (Fabaceae) }\end{array}$ & $\begin{array}{l}\text { South Andaman } \\
\text { (A. \& N. Islands) }\end{array}$ & Jan. 2002 & $\begin{array}{l}\mathrm{A}_{2,4,6,9-11}, \mathrm{~B}_{1-3,6,7,14,}, \mathrm{C}_{1}, \\
\mathrm{D}_{8,}, \mathrm{E}_{6,8,9,}, \mathrm{G}_{2,3,}, \mathrm{H}_{1}, \mathrm{~J}_{2,}, \mathrm{~L}_{1}, \mathrm{R}_{1}\end{array}$ & - \\
\hline $\begin{array}{l}\text { Cynometra ramiflora } \mathrm{L} . \\
\text { (PX) } \\
\text { (Fabaceae) }\end{array}$ & $\begin{array}{l}\text { South Andaman } \\
\text { (A. \& N) Islands }\end{array}$ & Mar. 2004 & $A_{2,4,9-11}, B_{1-3,6,7,14}, E_{9,}, G_{2,3}, H_{1}$ & - \\
\hline $\begin{array}{l}\text { Dalbergia candentensis (Dennst.) } \\
\text { Prain } \\
\text { (PX) } \\
\text { (Fabaceae) }\end{array}$ & $\begin{array}{l}\text { South Andaman } \\
\text { (A. \& N) Islands }\end{array}$ & Aug. 2002 & $\mathrm{G}_{2}$ & - \\
\hline $\begin{array}{l}\text { Derris elegans Benth. } \\
\text { (PX) } \\
\text { (Fabaceae) }\end{array}$ & $\begin{array}{l}\text { South Andaman } \\
\text { (A. \& N) Islands }\end{array}$ & Aug. 2002 & $\mathrm{G}_{2}$ & - \\
\hline $\begin{array}{l}\text { Dolichandrone spathacea (L.) } \\
\text { (K.) Schum. } \\
\text { (PX) } \\
\text { (Bignoniaceae) }\end{array}$ & $\begin{array}{l}\text { south Andaman } \\
\text { (A. \& N Islands) }\end{array}$ & Aug. 2002 & $\begin{array}{l}\mathrm{A}_{2,4,9-11}, \mathrm{~B}_{1-3,6,7,14}, \mathrm{C}_{1} \\
\mathrm{E}_{6,8,9,}, \mathrm{G}_{2,3,}, \mathrm{H}_{1}, \mathrm{~L}_{1}, \mathrm{R}_{1}\end{array}$ & - \\
\hline $\begin{array}{l}\text { Gelidiella acerosa (Forsk) } \\
\text { Feldman et Hamel. } \\
\text { (PL) } \\
\text { (Gelidiellaceae) }\end{array}$ & $\begin{array}{l}\text { Ramnad } \\
\text { (Tamil Nadu) }\end{array}$ & Nov. 2000 & $\begin{array}{l}A_{2,4,6,9-11}, B_{1-3,6,7}, C_{1,4,6} \\
E_{6,8,9}, G_{2,3,14}, H_{1}, L_{1}, R_{1}\end{array}$ & - \\
\hline
\end{tabular}


Table I. To be contd.

\begin{tabular}{|c|c|c|c|c|}
\hline $\begin{array}{l}\text { Heritiera fornes Buch.-Ham. } \\
\text { (PX) } \\
\text { (Sterculiaceae) }\end{array}$ & $\begin{array}{l}\text { South Andaman } \\
\text { (A. \& N Islands) }\end{array}$ & Dec. 2005 & NONE & - \\
\hline $\begin{array}{l}\text { Hypnea musciformis (Wulf.) } \\
\text { Lamour } \\
\text { (PL) } \\
\text { Hypneaceae }\end{array}$ & $\begin{array}{l}\text { Okha } \\
\text { (Gujarat) }\end{array}$ & Jan. 2000 & $\begin{array}{l}A_{2,4,9-11,} B_{1-3,6,7} \\
C_{4,6}, D_{8}, E_{8,9}, G_{3}, H_{1}, R_{1}\end{array}$ & - \\
\hline $\begin{array}{l}\text { Intsia bijuga (Colebr) O. Kutze } \\
\text { (PX) } \\
\text { (Caesalpiniaceae) }\end{array}$ & $\begin{array}{l}\text { South Andaman } \\
\text { (A. \& N Islands) }\end{array}$ & Mar. 2004 & $\begin{array}{l}A_{2,4,9-11} B_{1-3,6,7,14} \\
E_{9,} G_{2,3,14} H_{1}\end{array}$ & - \\
\hline $\begin{array}{l}\text { Kandelia candel (L.) Druce } \\
\text { (PX) } \\
\text { (Rhizophoraceae) }\end{array}$ & $\begin{array}{l}\text { Kendrapara } \\
\text { (Orissa) }\end{array}$ & Nov. 2003 & $\begin{array}{l}A_{2,74,6,9-11}, B_{1-3,6,7,14} \\
E_{9,} G_{2,3}, H_{1,2,} J_{9,10}\end{array}$ & - \\
\hline $\begin{array}{l}\text { Kandelia candel (L.) Druce } \\
\text { (LF) } \\
\text { (Rhizophoraceae) }\end{array}$ & $\begin{array}{l}\text { Kendrapara } \\
\text { (Orissa) }\end{array}$ & May 2000 & $\begin{array}{l}A_{2,4,9-11,} B_{1-3,6,7,} C_{1,4,6} \\
D_{8,} E_{6,8,9}, G_{2,3} L_{1}, R_{1}\end{array}$ & - \\
\hline $\begin{array}{l}\text { Kandelia candel (L.) Druce } \\
\text { (ST) } \\
\text { (Rhizophoraceae) }\end{array}$ & $\begin{array}{l}\text { Kendrapara } \\
\text { (Orissa) }\end{array}$ & May 2000 & $\begin{array}{l}A_{2,4,6,9-11,} B_{1-3,6,7}, C_{4,6}, D_{8}, E_{8} \\
G_{2,3}, H_{1}, L_{1}, N, R_{1}, Y_{1}\end{array}$ & - \\
\hline $\begin{array}{l}\text { Lumnitzera racemosa Willd. } \\
\text { (PX) } \\
\text { (Combrataceae) }\end{array}$ & $\begin{array}{l}\text { Chidambaram } \\
\text { (Tamil Nadu) }\end{array}$ & Jun. 2002 & $\mathrm{~A}_{6}, \mathrm{C}_{1}, \mathrm{E}_{6,8,9}, \mathrm{G}_{2,3}, \mathrm{H}_{1}, \mathrm{~L}_{1}$ & - \\
\hline $\begin{array}{l}\text { Lyngbya majuscula J. Ag } \\
\text { (PL) } \\
\text { (Oscillatoriaceae) }\end{array}$ & $\begin{array}{l}\text { Ramnad } \\
\text { (Tamil Nadu) }\end{array}$ & Jul. 2001 & $\begin{array}{l}A_{2,4,6,9-11,} B_{1-3,6,7}, D_{8}, E_{6,8,9} \\
G_{3,} H_{1,} R_{1}\end{array}$ & $\mathrm{D}_{8}$ (Antifilarial) \\
\hline $\begin{array}{l}\text { Monochoria vaginalis Phesl } \\
\text { (PL) } \\
\text { (Pontederiaceae) }\end{array}$ & $\begin{array}{l}\text { South Andaman } \\
\text { (A. \& N Islands) }\end{array}$ & Jan. 2002 & $\begin{array}{l}A_{2,4,9-11}, B_{1-3,6,7,14} \\
C_{1}, D_{8}, E_{6,8,9}, G_{3}, H_{1}, R_{1}\end{array}$ & - \\
\hline $\begin{array}{l}\text { Rhizophora apiculata (BI.) } \\
\text { (RB) } \\
\text { (Rhizophoraceae) }\end{array}$ & $\begin{array}{l}\text { South Andaman } \\
\text { (A. \& N Islands) }\end{array}$ & Nov. 2000 & $\mathrm{C}_{6}, \mathrm{G}_{14}, \mathrm{H}_{1,2}$ & $\begin{array}{c}\mathrm{G}_{14} \\
\text { ( Anti-trichomonas) }\end{array}$ \\
\hline $\begin{array}{l}\text { Rhizophora mucronata Lamk. } \\
\text { (LF) } \\
\text { (Rhizophoraceae) }\end{array}$ & $\begin{array}{l}\text { (Kendrapara) } \\
\text { (Orissa) }\end{array}$ & May 2000 & $\begin{array}{l}A_{2,4,6,9-11,} B_{1-3,6,7}, C_{1,4,6}, D_{8} \\
E_{6,8,9} G_{2,3}\end{array}$ & - \\
\hline $\begin{array}{l}\text { Rhizophora mucronata Lamk. } \\
\text { (ST) } \\
\text { (Rhizophoraceae) }\end{array}$ & $\begin{array}{l}\text { (Kendrapara) } \\
\text { (Orissa) }\end{array}$ & May 2000 & $\begin{array}{l}\mathrm{A}_{2,4,6,9-11}, \mathrm{~B}_{1-3,6,7}, \mathrm{C}_{1,4,6}, \mathrm{D}_{8} \\
\mathrm{E}_{6,8}, \mathrm{G}_{2,3}, \mathrm{~L}_{1}, \mathrm{~N}, \mathrm{R}_{1}, \mathrm{Y}_{1}\end{array}$ & - \\
\hline $\begin{array}{l}\text { Rhizophora apiculate BI. } \\
\text { (RT) } \\
\text { (Rhizophoraceae) }\end{array}$ & $\begin{array}{l}\text { South Andaman } \\
\text { (A. \& N Islands) }\end{array}$ & Dec. 2000 & $\mathrm{H}_{1,2}$ & - \\
\hline
\end{tabular}


Table I. To be contd.

\begin{tabular}{|c|c|c|c|c|}
\hline $\begin{array}{l}\text { Rhizophora lamarkii L. } \\
\text { (PX) } \\
\text { (Pontedpriaceae) }\end{array}$ & $\begin{array}{l}\text { Chidambaram } \\
\text { (Tamil Nadu) }\end{array}$ & Jun. 2002 & $\begin{array}{l}A_{2,4,6,9-11,} B_{1-3,6,7,14}, C_{5}, G_{2,3}, \\
J_{2,}, L_{1}, R_{1}\end{array}$ & - \\
\hline $\begin{array}{l}\text { Sonneratia griffithii Kurz. } \\
\text { (PX) } \\
\text { (Sonneratiaceae) }\end{array}$ & $\begin{array}{l}\text { South Andaman } \\
\text { (A. \& N. Islands) }\end{array}$ & Jan. 2002 & $\begin{array}{l}A_{2,4,9-11}, B_{1-3,6,7,14}, C_{5}, G_{2,3} \\
H_{1,} J_{2,} L_{1}, R_{1}\end{array}$ & - \\
\hline $\begin{array}{l}\text { Sonnerlatia griffithii Krrz } \\
\text { (PX) } \\
\text { (Sonneratiaceae) }\end{array}$ & $\begin{array}{l}\text { South Andaman } \\
\text { (A. \& N. Islands) }\end{array}$ & Mar. 2004 & NONE & - \\
\hline $\begin{array}{l}\text { UIva reticulata Forssk } \\
\text { (PL) } \\
\text { (Ulvaceae) }\end{array}$ & $\begin{array}{l}\text { Ramnad } \\
\text { (Tamil Nadu) }\end{array}$ & Nov. 2000 & $\begin{array}{l}A_{2,4,6,9-11}, B_{1-3,6,7}, C_{1,4,6}, D_{8} \\
E_{6,8,9}, G_{2,3}, H_{1}, L_{1}, N, Y_{1}\end{array}$ & - \\
\hline
\end{tabular}

A comprehensive biological screening of 33 marine plant species, occurring in Indian coasts, has been carried out. Many of these marine floras have been investigated chemically as well as biologically. However, the natural substances vary depending upon the ecological factors such as time and area of collection, etc. Interestingly, few of the marine floras showed biological activities that are not reported so far in literature.

\section{Acknowledgments}

Financial help from the Department of Ocean Development, New Delhi (India) is gratefully acknowledged. Experiments were performed as per the current laws of India.

\section{References}

Bhakuni D. S., Dhar M. L., Dhar M. M., Dhawan B. N. and Mehrotra B. N. (1969) Screening of Indian plants for biological activity: Part II, Indian J Exp Biol, 7: 250262.

Bhakuni D. S., Dhar M. L., Dhar M. M., Dhawan B. N., Gupta B. and Srimal R. C. (1971) Screening of Indian plants for biological activity: Part III, Indian J Exp Biol 9: 91-102.
Bhakuni D. S., Dhawan B. N., Garg H. S., Goel A. K., Mehrotra B. N., Srimal R. C. and Srivastava M. N. (1992) Bioactivity of marine organisms Part VI Screening of some marine flora from Indian Coasts, Indian J Exptl Biol, 30: 512-517.

Dhar M. L., Dhar M. M., Dhawan B. N., Mehrotra B. N. and Ray C. (1968) Screening of Indian plants for biological activity: Part I, Indian J Exp Bio, 6: 232-247.

Dhawan B. N., Garg H. S., Goel A. K., Srimal R. C., Srivastava M. N. and Bhakuni D. S. (1993) Bioactivity of marine organisms Part VII-Screening of some marine fauna from the Indian coasts, Indian $J$ Exptl Biol, 31: 505-510.

Dhawan B. N., Patnaik G. K., Rastogi R. P., Singh K. K. and Tandon J. S. (1977) Screening of Indian plants for biological activity: Part VI, Indian J Exp Biol. 15: 208219.

Kamat S. Y., Solimabi W., Naqvi S. W. A., Fernandes L., Reddy C. V. G., Bhakuni D. S. and Dhawan B. N. (1981) Bioactivity of the extracts from some marine animals of the Indian coast, Mahasagar, 14: 117-122.

Kamat S. Y, Wahidulla S., D'Souza L., Naik C. G., Ambiye V., Bhakuni D. S., Goel A. K., Garg H. S. and Srimal R. C. (1992) Bioactivity of marine organisms VI- 
Antiviral evaluation of marine algal extracts from the Indian coast, Bot Mar, 35: 161-164.

Kamat S. Y., Wahidulla S., Naik C. G., D'Souza L., Jayasree V., Ambiye V., Bhakuni D. S., Goel A. K., Garg H. S. and Srimal R. C. (1991) Bioactivity of marine organisms part III, Screening of some marine algae of the Indian coast for biological activity, Mahasagar, 24: 5357.

Lakshmi V., Goel A. K., Srivastava M. N. and Reghubir R. (2006a) Bioactivity of marine organisms: Part X, Screening of some marine fauna of indian coasts, Indian J. Exp. Biol, 44:754-758.

Lakshmi V., Goel A. K., Srivastava M. N., Kulshreshtha D. K. and Raghubir R. (2006) Bioactivity of marine organisms: Part IX, Screening of some marine flora of Indian coasts, Indian J Exp Biol, 44, 137-141.

Naik C. G, Kamat S. Y., Parameshwaran P. S., Das Bhattacharya J., Ramani P., Bhakuni D. S., Goel A. K., Jain S. and Srimal R. C. (1989) Bioactivity of marine organisms: Part IV, Screening of some marine animals from the indian coast, Mahasagar, 22: 99-104.
Naik C. G., Kamat S. Y., Parameshwaran P. S., Das B., Patel J, Ramani P., Bhakuni D. S., Goel A. K., Jain S. and Srimal R. C. (1990) Bioactivity of marine organisms: Part-V, Screening of some marine fauna from the Indian coast, Mahasagar, 23: 153-157.

Naqvi S. W. A., Solimabi W., Kamat S. Y., Fernandes L, Reddy C. V. G., Bhakuni D. S. and Dhawan B. N. (1980) Screening of some marine plants from Indian coast for biological activity, Bot Mar, 24: 51-55.

Siddhanta A. K., Mody K. H., Ramvavat B. K., Chauhan V. D., Garg H. S., Goel A. K. Doss Mj, Srivastava MN, Patnaik G. K. and Kamboj V. P. (1997) Bioactivity of marine organisms: Part VII, Screening of some marine flora of Western coast of India, Indian J Exp Biol, 35: 638-643.

Received : April 01, 2009;

Accepted : November 04, 2009 